
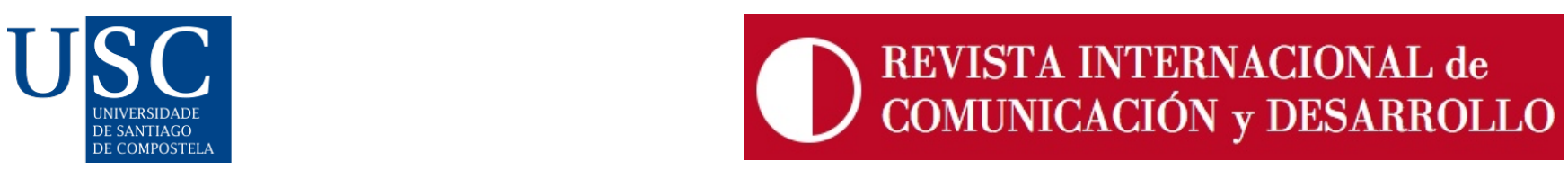

Revista Internacional de Comunicación y Desarrollo (RICD), 4(15), 2021. ISSN-e: 2386-3730

https://doi.org/10.15304/ricd.4.15.8180

Proyectos

\title{
InMedia: una metodología para el audiovisual participativo europeo con orientación inclusiva
}

\author{
Tamara Valladares ${ }^{1, a,{ }^{*}}$, Raquel Mariño Fernández ${ }^{2, b,{ }^{* *}}$, Marcelo Martínez Hermida ${ }^{3, c, * * *}$ \\ ${ }^{1}$ Universidade de Santiago de Compostela, España \\ ${ }^{2}$ Universidade de Santiago de Compostela, España \\ ${ }^{3}$ Universidade de Santiago de Compostela, España \\ a tamara.devera@usc.es b raquel.marino@usc.es c marceloantonio.martinez@usc.es
}

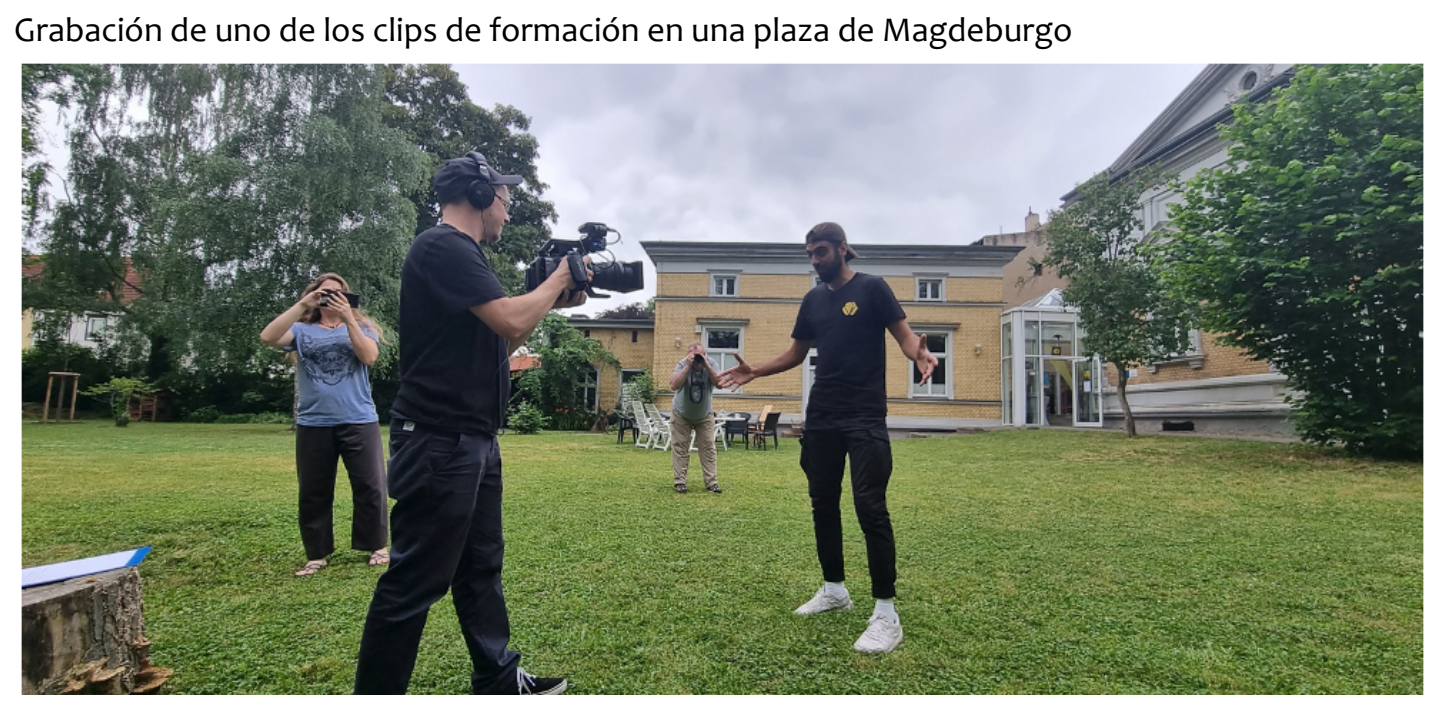

\section{EL PROYECTO EN BREVE}

InMedia $^{1}$ es un proyecto del programa Erasmus + de cooperación para la innovación y las buenas prácticas entre asociaciones estratégicas que pronto llegará a su primer año de desarrollo. El proyecto cuenta con treinta meses para alcanzar sus propósitos. Su principal objetivo es poner en marcha un trabajo activo entre medios de comunicación participativos y otros agentes europeos del audiovisual y la comunicación. Un proyecto orientado con y para personas afectadas por la exclusión social, según se plantea en su memoria inicial.

La formación de los operarios y voluntarios de las entidades socias del proyecto en el audiovisual participativo con orientación inclusiva es el proceso que articula y motiva la iniciativa InMedia.

Un total de seis socios participan en este proyecto de 30 meses de duración: Teleduca, en Barcelona, la Fédération de l'audiovisuel participatif —en la que participan unas 40 asociaciones y 
televisiones de Francia-, y el Offener Kanal, en Magdeburgo, pertenecen al llamado sector de los medios participativos. Estos tres socios, junto a la Universidade de Santiago de Compostela, han trabajado conjuntamente en los últimos años en el proyecto Media Fellow, también atento a los intercambios formativos sobre el audiovisual participativo (Breuzin y Dessset, 2017; González y Martinez, 2020).

En InMedia otras entidades como FilmForum, en Varsovia, o la Universidad „Lucian Blaga“ de Sibiu (LBUS), en Rumanía, se han unido y comprometido con la iniciativa. La asociación FilmForum tiene como propósito la educación de cineastas y destinatarios de la producción independiente de cortometrajes. Su empeño se centra en la creación de talleres y festivales de cine en Polonia y, para ello, colabora asiduamente con otros países del norte de Europa. LBUS es una pequeña universidad del centro de Rumanía con estudios de comunicación social y periodismo. A partir de este proyecto LBUS ha implementado sus actividades respecto del audiovisual, la comunicación participativa y la inclusión social con sus estudiantes de periodismo y pedagogía, con sus profesores y con profesionales de los medios locales de su ciudad. La participación de estas nuevas entidades como socias en InMedia plantea, como veremos, nuevos retos de adaptación respecto de la aplicabilidad de las formas de gestión y formación de estas entidades para con el audiovisual participativo.

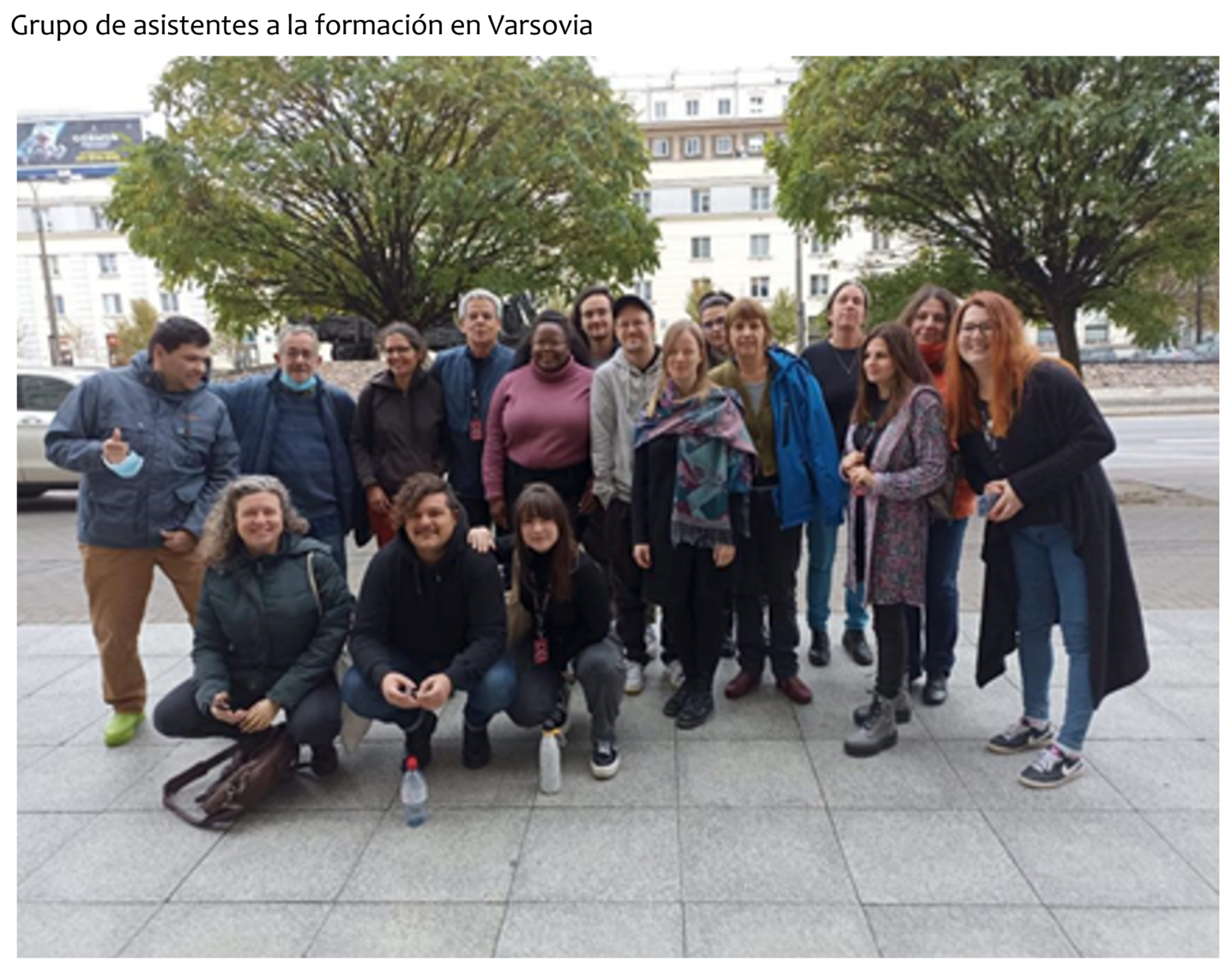

\section{PROCESOS, PROCEDIMIENTOS Y METODOLOGÍAS}

Los medios de comunicación comunitarios o participativos, como forma de medios de comunicación de la sociedad civil, comparten visibilidad con las personas y sus preocupaciones y, 
por tanto, tienen la misión de activar la participación de los individuos y los grupos sociales. En 2008, el Parlamento y el Consejo de Ministros de la UE declararon que los medios participativos son una parte importante del sistema democrático de medios de comunicación y pidieron a los Estados miembros que los reforzaran. A la fecha, en alguno de los países donde actúan los socios de InMedia (como por ejemplo España) todavía no se han satisfecho tales expectativas de la UE, entre otras cosas por las presiones que ejercen las grandes empresas de medios que conciben a la sociedad civil como simple consumidora de contenidos. Para asumir compromisos reales podría ser oportuno considerar en la reflexión común que InMedia pueda llevar a cabo, el concepto de comunidades de alta intensidad (Rocha, 2018), los procesos de resignificación identitaria (Figàs, 2020), la visibilización de los conflictos a través de festivales de cine (Martínez y Baumhardt, 2020) o la relectura de los procesos de concientización (Freire, 2017), entre otros que, en el laboratorio social latinoamericano, se han potencializado desde hace más tiempo en la academia, desde el conocimiento popular y desde la propia sociedad civil en el marco de las resistencias al maldesarrollo y en la inclusión de grupos vulnerables a través de la comunicación audiovisual. Asimismo también debiera de ser motivo de reflexión en estos proyectos la trayectoria diversa del propio concepto de audiovisual participativo, comparativamente, desde las prácticas que las entidades han desarrollado en dichos proyectos (Montero y Moreno, 2020).

La formación en participación e inclusión de los operarios y voluntarios que trabajan o que requieren experiencias de intercambio de procedimientos participativos e inclusivos, se ha entendido, primero en Media Fellow, y ahora en InMedia, como un proceso integrador y de gran relevancia para sustentar y consolidar las metodologías participativas o la orientación inclusiva con enfoque de derechos

Para el desarrollo de esta formación dirigida a los operarios y voluntarios de las propias entidades socias se ha asumido por parte de éstas la necesidad de consolidar una red local de beneficiarios y colaboradores territoriales que participen con sus preocupaciones y experiencias en los aprendizajes de los trabajadores de los medios. Que además visibilice el propósito de las entidades socias para con la sociedad civil a la que se deben y, al tiempo, las haga accesibles a grupos y personas que necesiten un altavoz o de sus conocimientos y recursos técnicos para expresarse socialmente.

En el proyecto Media Fellow los socios desplegaron algunas de las metodologías y técnicas que poseían como referencia para reconocer sus procedimientos habituales en el audiovisual participativo: el compagnonnage (Breuzin y Desset, ibid), la educomunicación (Mayugo, 2015) o el aktive medienarbeit (Tulodziecki, 1997; Niesyto, 2003; Schell, 2003; Mix, 2010) -sobre todo esta última metodología como orientación sugerida del audiovisual participativo en la memoria inicial del proyecto InMedia-.

En ambos proyectos la Universidade de Santiago de Compostela (USC) ha ejercido como recopiladora de los procedimientos que los otros socios debieran poner en valor en los aprendizajes y como proponedora de mejoras en la formación practicada para con los trabajadores y voluntarios asistentes. En Media Fellow activó encuestas y entrevistas a formadores y formandos sobre la satisfacción por la formación recibida, glosarios conceptuales como guía para la reflexión en las entidades participantes, ha elaborado informes de observación participante y participado en y de la formación, así como dispuesto técnicas de recogida de datos para los socios respecto de cartografías y técnicas relacionales. De otra parte, ha ido recogiendo y analizando algunos de los registros audiovisuales de las asambleas participativas que se han producido durante la formación, generado galerías de fotos y clips resúmenes sobre el proyecto Media Fellow, en particular. 
InMedia aprovecha la trayectoria instrumental y de análisis desplegada por la USC en Media Fellow, afinando en esta ocasión los métodos de recogida y análisis mediante cuestionarios y priorizando los objetivos del proyecto respecto de las entrevistas a entidades, colaboradores de las redes locales e implicados en la formación participativa. De otra parte, para el proyecto en curso, ha realizado una recogida de referencias y creado materiales de apoyo a los socios sobre el enfoque de derechos en prácticas inclusivas de grupos y personas en riesgo de exclusión social.

Se trata de una metodología que, desde la USC, tiene la intención de hacer comprensible la diversidad de prácticas de gestión y de producción que sobre la formación en audiovisual participativo despliegan los socios, por otra parte también diversos como entidades y estructuras, con marcos de actuación y redes colaboradoras asimismo diferentes Por tanto es esta una metodología compleja que hay que hacer sencilla para que sea utilizada en el día a día por los trabajadores y voluntarios de estas entidades, de manera que las redes locales de instituciones, asociaciones, personas y grupos vulnerables puedan beneficiarse finalmente.

La orientación inclusiva que debe de aportar InMedia en próximos aprendizajes se encontrará además con otras complejidades que, como pudimos vivir en Media Fellow, son altamente enriquecedoras en el aprendizaje operario y personal como el asumir la tolerancia y entender la interculturalidad necesarias a la hora de desarrollar audiovisual participativo entre trabajadores y voluntarios de los distintos territorios europeos. La dificultad de que los trabajadores y voluntarios puedan aplicar lo aprendido en sus entidades, en sus narraciones o en sus relaciones con grupos en riesgo de exclusión social, asoman en la formación como un reto a despejar más pronto que tarde en el marco del proyecto. Habrá que disponer de protocolos de acceso, de técnicas relacionales y hojas de ruta comunes y particulares, según las prácticas que asume cada socio en el día a día.

\section{LA FORMACIÓN EN INMEDIA: DE MAGDEBURGO A VARSOVIA}

En este primer avance del proyecto, para finalizar esta presentación sobre InMedia, recogemos la evaluación realizada por los formandos primero en las sesiones de Magdeburgo, del 21 al 25 de junio de 2021, a cargo de OK Magdeburg, Y, en segundo lugar, detallamos más concretamente las actividades, las estrategias compartidas y una primera aproximación de la evaluación de la formación realizada entre los días 2 y 6 de noviembre de 2021 en Varsovia, y que corrió a cargo de FilmForum. 
Sesión formativa en Magdeburgo

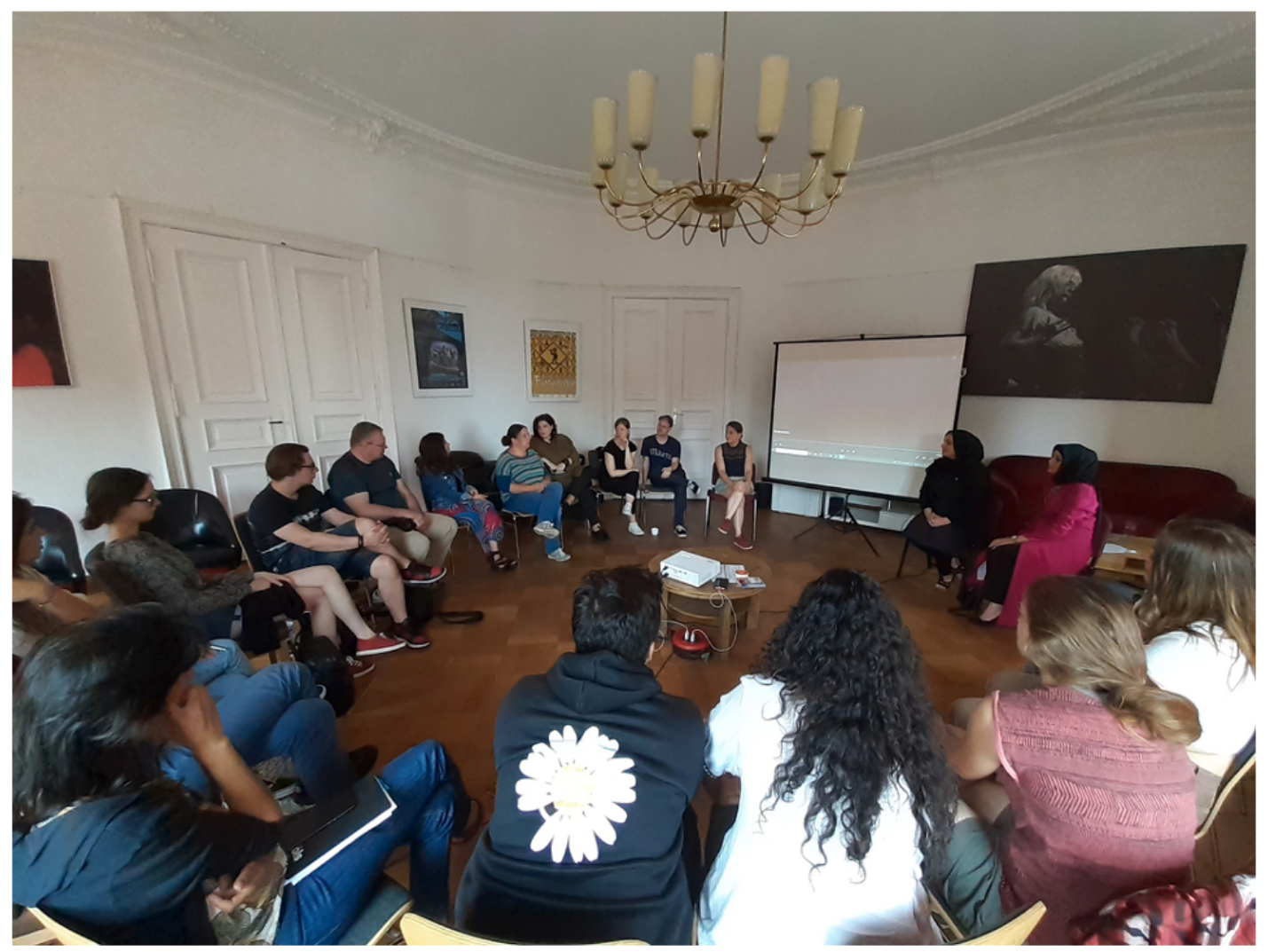

\subsection{Evaluación de las sesiones formativas en OK Magdeburg}

Entre los valores positivos de la formación impartida en OK Magdeburg destacar la satisfacción general de los participantes, que, en general, ha sido notablemente valorada.

También han sido valoradas en positivo las formadoras, las experiencias y las diversas piezas audiovisuales compartidas por aquellas en las sesiones de formación, así como la posibilidad de crear piezas audiovisuales integrando o implicando en ellas a colectivos en situación de vulnerabilidad o exclusión social.

De otra parte, los participantes en la formación valoran muy positivamente la oportunidad de crear redes de colaboración a través de las actividades que propone la formación. Por ello la gestión y organización de la formación reciben una buena calificación de los alumnos implicados.

No obstante, si profundizamos en las necesidades que los aprendices refieren sobre estas sesiones podemos detectar la urgencia de una mejora en la aplicabilidad, tanto de los contenidos abordados, como de los productos audiovisuales compartidos en la formación, hacia el trabajo diario que desarrollan comúnmente en las entidades de las que proceden. Es preciso mejorar, inciden los participantes, en la participación, la interculturalidad y la inclusión, e interactuar más con los grupos vulnerables de sus territorios. Incentivar, por tanto, una mayor participación en este tipo de eventos por parte de los profesionales del ámbito del trabajo social, así como de otros ámbitos profesionales tales como la psicología, la pedagogía o la educación social, parece aconsejable. 
Consideran necesario mejorar el uso compartido de los materiales empleados durante el proceso formativo. Y juzgan necesario lograr una mejora en la distribución temporal y cumplimiento horario de las sesiones planificadas en el programa. Lo que lleva añadido el deseo manifiesto de que se destine un mayor tiempo a las actividades para la realización de las piezas documentales.

Respecto de aquellas cuestiones detectadasobservadas que consideran necesario mejorar en futuras acciones, los alumnos listan las siguientes acciones:

- Identificar y mejorar el grado de accesibilidad y apoyos inclusivos presentes en los entornos laborales y mediáticos de los que proceden las personas operarias, valorando los recursos y obstáculos físicos y estructurales disponibles o no en los edificios de las entidades, así como las herramientas de comunicación utilizadas, de manera que sean verdaderamente inclusivas.

- Para ello hay que lograr que las entidades colaboradoras y sus operarios y operarias realicen mapeos de accesibilidad en sus espacios de trabajo. Y de usabilidad inclusiva en aquellos contenidos que producen.

- Mejorar la formación en competencias y dominios de los operarios y operarias que trabajan en entidades mediáticas en materia de inclusión, perspectiva de interculturalidad y metodologías participativas con aquellos colectivos en situación de exclusión social o vulnerabilidad presentes en sus comunidades.

- Crear más seminarios de reciclaje y de formación continua que permitan ampliar, mejorar y/o recualificar a sus operarios y operarias en las metodologías participativas, perspectivas de inclusión y enfoques de interculturalidad que se promueven desde InMedia.

- Crear protocolos y guías de actuación para incentivar y gestionar la diversidad del voluntariado, haciéndolo más atractivo y beneficioso para incluir a las personas que forman parte de estos colectivos.

- Conocer las necesidades y demandas reales sobre inclusión y diversidad intercultural mediática (agenda inclusiva) que manifiestan las personas que forman parte de estos colectivos en situación de exclusión y vulnerabilidad.

- Fomentar la integración y participación real de los colectivos vulnerables o en situación de marginación en los procesos de toma de decisiones, en los procesos creativos y en la generaciónproducción de piezas audiovisuales.

- Visibilizar y compartir voz protagonista con estos colectivos para que narren en primera persona sus experiencias, que tengan espacios desde los que compartir valiosas opiniones desde otros puntos de vista, ofrecerles oportunidades para que compartan sus vivencias en piezas audiovisuales que reflejen la verdadera realidad en la que viven.

- Incentivar la creación de nuevas redes de trabajo colaborativo y comunidades participativas, integrando en las mismas a sujetos pertenecientes a estos colectivos vulnerables, así como a entidades y asociaciones que destaquen por sus buenas prácticas en materia de inclusión y gestión de la diversidad.

- Ampliar y fortalecer las redes que se establecen con los socios del proyecto InMedia para que se consoliden como verdaderas comunidades inclusivas y participativas.

- Adaptar la aplicabilidad de las sesiones y actividades formativas a los desempeños laborales reales de los puestos que ocupan los operarios y operarias que acuden a 
formarse, para que puedan incorporarlas y proyectarlas en su trabajo diario dentro de las empresas.

- Dar a conocer el potencial didáctico y posibilidades de usos y mejoras cualitativas que aportan estas experiencias formativas a la creación de piezas audiovisuales con perspectiva de inclusión.

- Hacer un mejor uso de los tiempos y de los talleres prácticos que se ofrecen en estas sesiones formativas, dado que los procesos creativos, inclusivos e interculturales son acciones que precisan una mayor dedicación para ser contextualizadas por parte de los colectivos implicados en los mismos.

\subsection{Las sesiones formativas en FilmForum}

La formación se encuadró dentro de uno los eventos cinematográficos que organizan, el Cinemaforum, desarrollándose a la par que las proyecciones y a las que los participantes de InMedia pudieron asistir libremente. Un total de doce participantes y cinco personas del grupo de planificación del proyecto se encontraron en la capital polaca para compartir estos días de aprendizaje

En líneas generales las actividades desarrolladas en el programa consistieron en visitas a centros profesionales en el desarrollo de formación en audiovisual (Warsaw Film School, Andrzej Wajda Film Culture Centre, o la Kinoteka); charlas con diferentes creadores de contenido audiovisual sin ánimo de lucro (Marek Pawlikowski y Karim Kourani de la asociación socia de Filmforum, Kinographe), con un grupo LGTB+ de voguing y con el director del festival de LGTB+ Film Festival, comunidad que actualmente sufre de vejaciones y discriminaciones graves en el contexto polaco; actividades de presentación y conocimiento mutuo de los socios del proyecto, así como de intercambio de diversas experiencias participativas y de cariz inclusivo desarrolladas en sus entidades. Y por último, asistencia a las proyecciones del festival. 


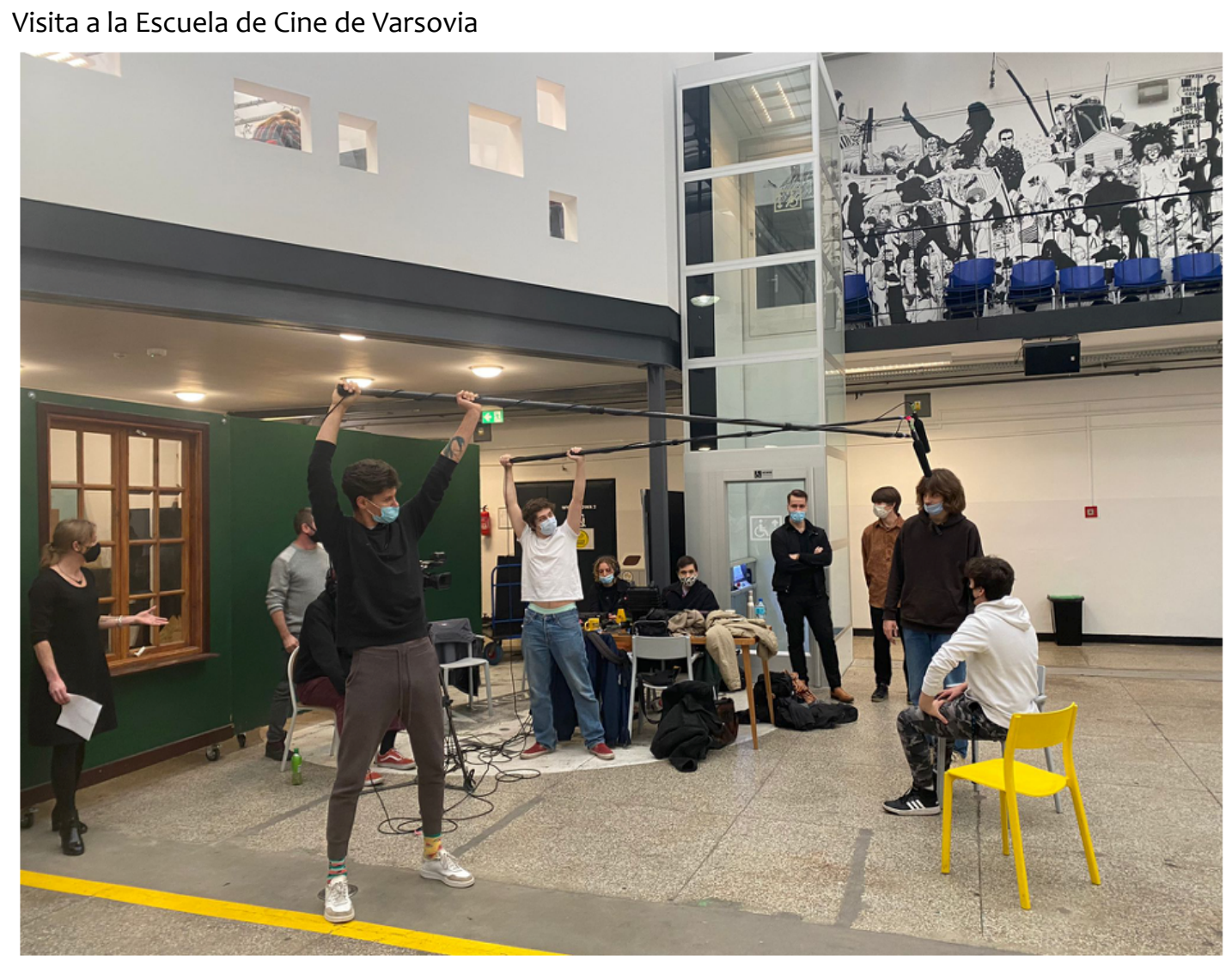

Uno de los aspectos clave de la formación fueron los espacios de conocimiento mutuo que se desarrollaron en la primera sesión. En este momento las diferentes organizaciones socias del proyecto pudieron dar a conocer sus proyectos y sus perspectivas sobre que entienden por trabajo audiovisual comunitario e inclusivo.

En el caso de Offener Kanal presentaron su proyecto "Magdeburg Moritzplatz".

https://www.youtube.com/watch?v=qMeihBxtVcA\&list=PLCaiAH7_5REKdqW8H7btnPwB0uS IQcR6y.

Moritzplatz, es un barrio de Magdeburgo caracterizado por los conflictos interculturales e integeneracionales, así como por las bajas rentas de la población. El proyecto de Offener Kanal consiste en la realización de una serie contando con la juventud del barrio, en conjunto basándose en sus vivencias personales construyen el guion, diálogos de la serie y desarrollan los personajes. Son los propios jóvenes los que protagonizan la serie, la graban y colaboran en el proceso de montaje.

Los representantes de la Fédération de l'audiovisuel participatif, explicaron el proceso de creación de canales participativos de comunicación independientes a los mainstream como es el caso de "Mo Tv" o "Tele Mouche".

Y Teleduca dio a conocer sus proyectos de Educomunicación con jóvenes, que tratan de utilizar el audiovisual como medio para crear comunidad desde una perspectiva participativa. Uno de los aspectos más interesantes de esta presentación fue que uno de los jóvenes participantes de uno de sus proyectos de Educomunicación fue el que expuso la experiencia, ahora como voluntario de la organización. 
https://www.youtube.com/channel/UCHf5qWU_1Cn40WBr2eytKpA/videos

En el transcurso de la semana de sesiones formativas se organizó un encuentro (jueves 4 de noviembre) entre las personas participantes del proyecto (asistentes a las formaciones), con el grupo directivo y el grupo organizador del encuentro para evaluar las actividades realizadas hasta el momento desde un enfoque inclusivo. El encuentro se desarrolló de manera participada, a modo de asamblea en el que cada una de las personas participantes tuvieron tiempo y espacio para comunicar, de manera asertiva y colaborativa los aspectos fuertes y débiles de la formación hasta ese momento. El objetivo era mejorar el transcurso de la formación y de las futuras formaciones en Francia y España.

Algunas de las valoraciones más repetidas entre los participantes fue el interés que suscitó conocer el funcionamiento de las plataformas de cine como es el caso de FilmForum y su trabajo reconocido en Polonia en la programación y desarrollo de festivales de cine. También les resultó positivo el conocer instituciones de referencia en la creación de audiovisual como fue el caso de la visita a la Warsaw Film School, el Andrej Wajda Film Culture Centre y la Kinoteka, en el Palace of Culture and Science, edificio característico de Varsovia. Así como poder acceder de manera gratuita a todas las instalaciones del festival. Les pareció de utilidad para continuar mejorando sus capacidades técnicas a la hora de elaborar contenidos audiovisuales atractivos a nivel estético y conceptual, muy interesante para hacer llegar mejor sus mensajes al gran público.

Aún así, expresaron sus inquietudes acerca de que, en la formación, los organizadores no tuvieron tan en cuenta la necesidad de trabajar con mirada inclusiva para abrir el espacio de las organizaciones de creación y contenido audiovisual participativo a grupos vulnerables. En el caso del socio FilmForum, se encuentran aún en proceso de adaptar sus estructuras a procedimientos y contenidos más inclusivos, son una gran corporación con capital y experiencia en el sector del audiovisual pero nuevos en el trabajo del audiovisual más participativo e inclusivo.

Esta evaluación les sirvió para entender, con más matices, como trabajan los otros socios y qué se espera del proyecto, mostrando interés en el tema y adaptando las siguientes sesiones de formación a las expectativas de los participantes. Así como tomando en cuenta las valoraciones para ir incluyendo estos aspectos en sus festivales.

Los participantes buscan trabajar más en grupo, buscan crear redes de confianza para la creación de proyectos comunes que aúnen a diferentes países europeos en la creación de proyectos audiovisuales que tengan a todos y a todas en cuenta.

En concreto de aquellas personas que, por razones de discriminación social de diferente tipo (étnico, político, económico, social, de género o de edad...) no tienen acceso al conocímiento, métodos o herramientas para desarrollar sus historias y dar a conocer sus proyectos.

Para ello necesitan más formaciones prácticas en el que se muestren métodos de trabajo participativo e inclusivo en el audiovisual, para no caer en miradas paternalistas y ofrecer realmente espacios de colaboración seguros a estos colectivos.

\section{REFERENCIAS BIBLIOGRÁFICAS}

Breuzin, A. y Desset, T. (2017). Media Fellow: compartiendo prácticas por y para los medios participativos a escala europea. Revista Internacional de Comunicación y Desarrollo, 2(7), 131-134. https://revistas. usc.gal/index.php/ricd/article/view/4480 
Figàs, M. (2020). Normativas audiovisuales colectivas como vehículo de resignificación identitaria: implementando el video participativo con jóvenes guatemaltecos. Revista Internacional de Comunicación y Desarrollo, 3(11), 80-91. https://doi.org/10.15304/ricd.3.11.6381

Freire, P. (2017). Conscientização. Cortez Editora.

González, A. y Martínez, M. (2020). Metodología de la formación para el audiovisual participativo europeo: Media Fellow. Revista Internacional de Comunicación y Desarrollo, 3(13), 130-137. https://doi.org/10 $.15304 /$ ricd.3.13.7363

Martínez, A. y Baumhardt, F. (2020). Participatory video at the UN Global Migration Film Festival: engaging displaced indigenous Venezuelans in filmaking at a setter in Brazil. Revista Internacional de Comunicación y Desarrollo, 3(11), 67-79. https://doi.org/10.15304/ricd.3.11.6406

Martínez, M. y Ogando, L. (2019). Av Coruña Común: breve cartografía do audiovisual participativo coruñés e galego. Revista Internacional de Comunicación y Desarrollo, 3(10), 159-165. https://www.revistas.u sc.gal/index.php/ricd/article/view/6179

Mayugo, C. (2015). La confluencia entre Educomunicación y Comunicación Comunitaria en las prácticas de creación del Audiovisual Participativo [Tesis doctoral]. Universidade de Santiago de Compostela.

Mix, S. (2010). Aktive Medienarbeit mit Jugendlichen. Theoretische Grundlagen und praxisbezogene Beispiele. [Tesis]. Fachhochschule Dortmund,

Montero, D. y Moreno, J.M. (2020). Explorando el campo del conocimiento del video participativo. Un recorrido por las principales aportaciones teóricoprácticas. Revista Internacional de Comunicación y Desarrollo, 3(11), 92-108. https://doi.org/10.15304/ricd.3.11.6345

Niesyto, H. (2003). (hg.) VideoCulture. Video und interkulturelle Kommunikation. Kopaed.

Rocha, C.A. (2018). El Audiovisual participativo y la ciudadanía de alta intensidad en Cazuca (Colombia). Revista Internacional de Comunicación y Desarrollo, 2(9), 42-56. https://doi.org/10.15304/ricd.2.9.52 $\underline{14}$

Schell, F. (2003). Aktive Medienarbeit mit Jugendlichen. Kopaed.

Tulodziecki, G. (1997). Medien in Erziehung und Bildung. Grundlagen und Beispiele einer handlungs und entwicklungsorientierten Medienpädagogik. Klinkhardt.

\section{Notas}

* Tamara Valladares de Vera es magister en Investigación de la Educación, Diversidad Cultural y Desarrollo Comunitario. Es licenciada en Educación Social por la USC, y especialista en Educación para la Ciudadanía Global. Profesora del área de Teoría e Historia de la Educación, está adscrita al grupo de investigación Terceira Xeración de la USC.

Contacto: tamara.devera@usc.es

** Raquel Mariño Fernández es doctora en Ciencias de la Educación y licenciada en Psicopedagogía. Profesora del Área de Didáctica y Organización Escolar y miembro del grupo de investigación Gefil de la USC su trayectoria formativa se relaciona con el género y la diversidad, además de con la Formación Profesional y para el Trabajo. Contacto: raquel.marino@usc.es

*** Marcelo Martínez Hermida es doctor en Ciencias de la Comunicación por UCM y licenciado en Periodismo por UAB. Coordinador del grupo de investigación Cidadanía e Comunicación, es profesor titular en las carreras de Periodismo y de Comunicación Audiovisual y director de la Revista Internacional de Comunicación y Desarrollo, en USC.

Contacto: marceloantonio.martinez@usc.es

1 El equipo de trabajo en InMedia está compuesto por profesores e investigadores de varios grupos de investigación de USC. A la fecha, en la recogida y análisis de datos para InMedia, han participado además: Jésica Núñez (Esculca), Esther Olveira y Silvana Longueira (TeXe). 